AperTO - Archivio Istituzionale Open Access dell'Università di Torino

\title{
Thermoresponsive Polymer Grafted Porous Silicas as Smart Nanocarriers
}

\section{This is the author's manuscript}

Original Citation:

\section{Availability:}

This version is available http://hdl.handle.net/2318/1692006

since 2019-02-12T17:04:05Z

Published version:

DOI:10.1071/CH18229

Terms of use:

Open Access

Anyone can freely access the full text of works made available as "Open Access". Works made available under a Creative Commons license can be used according to the terms and conditions of said license. Use of all other works requires consent of the right holder (author or publisher) if not exempted from copyright protection by the applicable law. 


\title{
Thermoresponsive Porous Silica as Smart Pharmaceutical Nanocarriers
}

\author{
Sushilkumar A. Jadhav*, Dominique Scalarone \\ Department of Chemistry and Nanostructured Interfaces Surfaces (NIS) Research Centre \\ University of Torino, 10125, Torino, Italy. \\ E-mail: sushil.unige@ gmail.com
}

\begin{abstract}
Porous silica particles grafted with various stimuli-responsive polymers are investigated with great interest for their use as smart pharmaceutical nanocarriers in advanced drug delivery systems (DDS). In particular, porous silica particles grafted with thermoresponsive polymers that exhibit thermally triggered on/off gating mechanisms have shown improved performance as hybrid DDS capable of controlling the release of different drugs in various mediums which resemble complex biological environments. In addition, the tuning of the drug release profiles as per requirements has proved possible with modifications to the porous core and the grafted thermoresponsive polymers. This highlight presents a brief discussion of basic preparation techniques and some recent significant developments in the field of thermoresponsive polymer grafted porous silica particles as smart pharmaceutical nanocarriers.
\end{abstract}

Keywords: porous silica particles, thermoresponsive polymers, hybrid silica particles, thermoresponsive silica, drug delivery 


\section{Introduction}

Porous silica particles are investigated with great interest as pharmaceutical nanocarriers in advanced drug delivery systems (DDS) [1-6]. In particular, organic groups and polymergrafted porous silica particles have shown better efficiency as DDS with respect to pristine particles. The efficiency and selective control over the drug release from porous nanocarriers can be further enhanced by grafting them with stimuli-responsive systems and smart polymers [7-9]. The grafting of smart polymers transforms simple porous particles into stimuli-controlled smart pharmaceutical nanocarriers. The stimuli-responsive polymer shell around the porous particles serves two purposes, first it helps to trigger the delivery of drug molecules by an external stimulus and second the polymer shell alters the surface properties of the bare particles preventing their aggregation. Porous silica particles exhibiting thermosensitive gating mechanisms are one of the most interesting and highly studied DDS [10]. Thermoresponsive polymers are a class of smart polymers and their physical properties are altered due to changes in temperature. These polymers exhibit a volume phase transition at their specific lower critical solution temperature (LCST). Above the LCST, the soluble hydrated polymer chains collapse to form insoluble hydrophobic globules, this phenomenon is called as the coil-to-globule transition and is reversible [1113]. Examples of the most common thermoresponsive polymers are poly(Nisopropylacrylamide) (PNIPAM) and its various copolymers such as poly(Nisopropylacrylamide-co-acrylamide) (poly(NIPAM-co-AA)), poly(N-isopropylacrylamideco-acrylic acid) etc., poly(N-vinyl caprolactam), poly(N-ethyl oxazoline), and poly(methyl vinyl ether)s etc. (refer to Fig. 1a for structures of some common thermoresponsive polymers). The grafting of thermosensitive polymers applies a pore-opening (on) poreclosing (off) mechanism to the porous particles by virtue of their transition at the LCST. The polymers in their extended coil conformation at below the LCST occupy most of the pore volume which prevents the diffusion of molecules loaded inside them. Instead, above 
the LCST the polymer chains collapse to form very compact hydrophobic globules that open the pores. This mechanism is controlled by changes in temperature (external stimulus, Fig. 1b). The thermosensitive gating mechanism allows triggering the drug release at elevated temperatures (above LCST) and at desired rates. The gating mechanism can also be reversed by adjusting the pore diameters or molecular weight and LCST/upper critical solution temperature (UCST) of the polymers. Post-synthesis surface grafting of thermoresponsive polymers by 'graft from' or 'graft to' methods on porous particles is carried out to prepare thermosensitive DDS. The well known alkoxysilane grafting protocol is used for the grafting of monomers, initiators, or directly preformed thermoresponsive polymers on porous silica particles [14]. Due to the inert nature of silica it does not interfere with the various surface initiated polymerization (SIP) techniques such as free radical polymerization or atom transfer radical polymerization (ATRP), reversible addition-fragmentation chain transfer (RAFT) etc. [15-17]. Apart from applying the gating mechanism, the polymer additionally helps in the internalization of particles (endocytosis) in cells by undergoing the thermoresponsive transition. The transition of the polymer at its LCST transforms the hydrophilic surface of the particles into a hydrophobic surface (Fig. 1c). As the hybrid particles at that temperature exhibit a hydrophobic surface they can be easily internalized by various cells. Almost a 20 -fold increase in cellular uptake was observed at elevated (above LCST) temperatures [18-20]. In addition to the alteration of surface properties the coil-to-globule transition of thermoresponsive polymers can be used to hide and reveal or mask and uncover active functionalities present (or purposely grafted) on the surface, and this in turn can be used to direct molecular and cell recognition phenomena.

\section{Recent Highlights}


Some recent reports in the literature present significant developments in the preparation of thermoresponsive smart nanocarriers based on porous silica particles for the delivery of drugs from various classes such as anti-cancer, anti-inflammatory, antioxidants, and proteins. These developments also include the synthesis of new multi-responsive copolymers, which generates complex double or triple responsive advanced smart nanocarriers. Yu et al. [21] recently reported a porous silica-based DDS grafted with a temperature and reactive oxygen species (ROS) responsive polymer (ROSP@mesoporous silica nanoparticles, MSNs) for delivery of the anticancer drug doxorubicin (DOX). The thermo andROSresponsive 4-(4,4,5,5-tetramethyl-1,3,2-dioxaborolan-2-yl)benzyl acrylate modified polymer was grafted on porous silica particles and controls the release of the drug. Hydrogen peroxide mediated detachment of the ROS species 4-(methylol) phenylboronic acid pinacol ester at elevated temperatures from the copolymer structure (Fig. 2) generates the poly(NIPAM-co-AA) copolymer which has a LCST at around $37^{\circ} \mathrm{C}$. The transition of the polymer at that temperature helps in releasing DOX by opening the pores. The hybrid particles showed good biocompatibility and internalization by cells. With the increased concentration ofDOXdue to its release with time the Hela cell viability was decreased to less than $30 \%$. Hegazy et al. [22] reported magnetic, reductive, and thermo triple-responsive nanocarriers based on magnetite-silica (core) thermo/pH responsive polymer (shell) nanoparticles. This is a classic example of how multiple responsive mechanisms can be grafted on porous silica particles. The loading and release of DOX mediated by all three stimuli mechanisms was reported where a significant difference in DOX release at low and elevated temperatures was observed. In comparison with the single responsive systems, such triple-responsive porous smart pharmaceutical carriers hold greater potential for controlling the drug delivery process in the complicated blood circulation and pathological environment. Although the work does not present any biological testing, it shows the stability of the hybrid magnetic thermoresponsive polymer 
grafted porous silica nanoparticles in physiological conditions (saline buffer). This is important to prove that the silica-based hybrid porous materials can remain intact during their use. Wu et al. [23] reported a dual ( $\mathrm{pH} /$ thermo) responsive DDS based on porous silica with a mixed polymer shell of PNIPAM and polyethylene glycol (PEG). The transition of PNIPAM chains above its LCST showed thermally triggered release of DOX from the porous particles at elevated temperatures while the PEG chains were found to provide a hydrophilic barrier and impart colloidal stability and in vivo longevity to the DDS. A very good internalization of the thermoresponsive polymergrafted particles by HCT-116 cells was observed by incubation for $12 \mathrm{~h}$ at $\mathrm{pH}$ 7.4. The cell cytotoxicity experiments using the drug (DOX)-loaded particles showed decreased cell viability (up to $40 \%$ ). Some other recent reports on thermoresponsive silica include the development of porous silica-PNIPAM or its copolymer-based hybrid DDS for the delivery of antiinflammatory drugs such as ibuprofen [24-26], antioxidants like rutin [27] and quercetin [28], and fluorescent molecules like fluorescein [29]. Most of these works point out the important roles of pore dimensions (volume/multimodality), PNIPAM-based copolymer composition, and molecular weight of the polymers in modulating the drug release profiles from the thermoresponsive silica particles. Importantly, these studies prove that desired control over the drug release in different environments is possible by selective modifications of the hybrid DDS. Theoretically, no or very low drug release by the thermoresponsive polymer-grafted particles is expected below the LCST of the polymer. Instead, a triggered higher release of drugs at elevated temperatures above the LCST is expected. However, this trend can be reversed. In the work by Guisasola et al. [29] the synthesis and testing of magnetic porous silica grafted with thermoresponsive polymeric gates with opposite controlled release behaviours was presented. They proved that the thermally triggered release profiles of fluorescein from the thermoresponsive copolymer based on NIPAM and N-hydroxymethyl acrylamide (NHMA) grafted porous particles 
depend upon the presence of cross-linked and not cross-linked (linear chains or brushes) polymers. A low release profile below the LCST $\left(42^{\circ} \mathrm{C}\right)$ of the polymer was observed for the cross-linked polymer-grafted particles. Instead, the release profile was reversed for the linear polymer-grafted particles which showed higher drug release below the LCST. In another recent report [27], it is proved that, not only the polymer shell but also the multimodal porous channel structure present on the porous silica particles can alter the drug release behaviour from porous silica-based thermoresponsive DDS. Such reversible thermoresponsive release behaviour can be of great use in therapies where a sustained release of drug is desired at higher temperatures instead of a triggered higher release. A recent study by $\mathrm{Yu}$ et al. [30] reported the loading and thermally triggered release of bovine hemoglobin $(\mathrm{BHb})$ protein from thermoreponsive porous silica particles. Proteins are big biomolecules and may not be incorporated inside the pores on conventional MCM41 or SBA-15 type porous silica nano or microparticles. For this purpose novel poreexpanded mesoporous silica nanoparticles (MSNs) with pore sizes of $11 \mathrm{~nm}$ were synthesised and grafted with thermoresponsive PNIPAM. PEGylation of the interior pore surface was done to avoid silica surface-protein interactions. A thermally triggered $\mathrm{BHb}$ release in physiological conditions was observed with significant difference in protein release below $\left(25^{\circ} \mathrm{C}\right)$ and above $\left(37^{\circ} \mathrm{C}\right)$ the LCST of the polymer (Fig. 3). This is a rare report where loading and release of protein from thermoresponsive porous silica was tested. The pore expansion in porous silica is possible due to various recently invented synthetic techniques which give the possibility of tuning the pore diameters [31]. The pore diameters and volumes can be increased to incorporate large molecules such as proteins. Related to this, a previous study by Tu et al. [32] showed the importance of tuning of the diameters of the pores on silica for the incorporation of big molecules like proteins. They investigated the encapsulation and release behaviour for seven model proteins named alactalbumin (LAC), ovalbumin (OVA), bovine serum albumin (BSA), catalase (CAT), 
haemoglobin (HB), lysozyme (LYS), and cytochrome (CYT) from large pore porous silica particles functionalized with 3-aminopropyltriethoxysilane or 3-[2-(2aminoethylamino)ethylamino]propyltrimethoxysilane (APMSNs and AEP-MSNs) (Fig. 3). The report by $\mathrm{Yu}$ et al. proved that further control over the release of proteins from expanded pore silica can be achieved by grafting with thermoresponsive polymers, and protein-silica surface interactions can be limited by grafting the pore walls with PEG chains.

\section{Conclusions and Future Challenges}

Due to very intense research in the last two decades on bare or simply functionalized porous silica particle based DDS, they have reached the advanced stages of testing such as clinical trials. Many aspects such as in vitro and in vivo drug release studies, biocompatibility, toxicity, biocompatibility, internalization by cells, and fate in vivo have been studied in detail [33]. Instead, the polymer or in particular the stimuli responsive polymer-grafted porous silica based DDS still have to go a long way before they reach the final stages of testing. Hence there is great scope for continued research for the improvement of thermoresponsive polymer-grafted porous silica nanocarriers. The future research on these materials will continue in the following main directions: 1) To design and synthesise an ideal DDS with a perfect thermosensitive gating mechanism where ideally complete blockage of drugs is possible below the LCST of the polymer. Although several new and more efficient thermoresponsive polymers and copolymers have been synthesized and tested to improve the performance of thermoresponsive nanocarriers, the approval of these new polymers for biomedical uses is a matter for separate discussion. 2) Extended detailed studies about the biocompatibility, toxicity, and internalization. Studies on the fate of the hybrid nanocarriers in vivo after drug release, that is either in vivo degradation of them or clearance from the body. There are no conclusive studies on this 
point reported to date. 3) In the case that porous thermoresponsive silicas will cross all the hurdles and come up as efficient DDS, reproducible and large scale synthesis methods for their production will be a challenge. The synthesis methods should be developed in such a way that they will guarantee the production of polymer-grafted porous silicas with desired properties for biomedical use. Considering the recent continued interest and advancements, thermoresponsive polymer-grafted porous silica materials have shown great potential for being the next generation smart pharmaceutical nanocarriers. The challenges related to further development of these materials are wide open and continued research in future will definitely address the present shortcomings and reveal much more interesting aspects of these materials.

\section{References}

1 J. Florek, R. Caillard, F. Kleitz, Nanoscale, 2017, 9, 15252-15277.

2 M. Vallet-Regí, F. Balas, D. Arcos, Angew. Chem. Int. Ed. 2007, 46, 7548-7558.

3 C. Argyo, V. Weiss, C. Bräuchle, T. Bein, Chem. Mater. 2014, 26, 435-451.

4 Baeza, D. Ruiz-Molina, M. Vallet-Regí, Expert Opin. Drug Deliv. 2017, 14, $783-$ 796.

5 S. A. Jadhav, Inorg. Chem. Front. 2014, 1, 735-739.

6 S. A. Jadhav, J. Nanomater, 2016, Article ID 1325174, DOI: 10.1155/2016/1325174.

7 Y. Song, Y. Li, Q. Xu, Z. Liu, Int. J. Nanomedicine, 2017, 12, 87-110.

8 Y. Zhao, J. L. Vivero-Escoto, I. I. Slowing, B. G. Trewyn, V. S.-Y. Lin, Expert Opin. Drug Deliv. 2010, 7, 1013-1029.

9 Y. Chen, H. Zhang, X. Cai, J. Ji, S. He, G. Zhai, RSC Adv., 2016, 6, 92073-92091.

10 M. Bathfield, J. Reboul, T. Cacciaguerra, P. Lacroix-Desmazes, C. Gérardin, Chem. Mater. 2016, 28, 3374-3384. 
11 D. Roy, W. L. A. Brooks, B. S. Sumerlin, Chem. Soc. Rev. 2013, 42, 7214.

12 Y. J. Kim, Y. T. Matsunaga, J. Mater. Chem. B, 2017, 5, 4307-4321.

13 A. Gandhi, S. O. Paul, K. K. Sen, Asian J. Pharm. Sci. 2015, 10, 99-107.

14 S. A. Jadhav, V. Brunella, D. Scalarone, Part. Part. Syst. Charact., 2015, 32, 417428.

15 Radhakrishnan, R. Ranjan, W. J. Brittain, Soft Matter, 2006, 2, 386-396.

16 L. Wu, U. Glebe, A. Böker, Polym. Chem. 2015, 6, 5143-518.

17 M. Müllner, J. Cui, K. F. Noi, S. T. Gunawan, F. Caruso, Langmuir, 2014, 30, 62866293.

18 F. Mastrotto, P. Caliceti, V. Amendola, S. Bersani, J. P. Magnusson, M. Meneghetti, G. Mantovani, C. Alexander, S. Salmaso, Chem. Commun., 2011, 47, 9846.

19 S. Behzadi, V. Serpooshan, W. Tao, M. A. Hamaly, M. Y. Alkawareek, E. C. Dreaden, D. Brown, A. M. Alkilany, O. C. Farokhzad, M. Mahmoudi, Chem. Soc. Rev., 2017, 46, 4218-4244.

20 M. Hei, J. Wang, K. Wang, W. Zhu, P. X. Ma, J. Mater. Chem. B, 2017, 5, $9497-$ 9501.

21 F. Yu, H. Wu, Y. Tang, Y. Xu, X. Qian, W. Zhu, Int. J. Pharm. 2018, 536, 11-20.

22 M. Hegazy, P. Zhou, G. Wu, L. Wang, N. Rahoui, N. Taloub, X. Huang, Y. Huang, Polym. Chem. 2017, 8, 5852-5864.

23 Q. Wu, Y. Hou, G. Han, X. Liu, X. Tang, H. Li, X. Song, G. Zhang, Nanomedicine, 2017, 12, DOI 10.2217/nnm-2017-0216.

24 L. García-Uriostegui, H. I. Meléndez-Ortiz, G. Toriz, E. Delgado, Mater. Lett. 2017, 196, 26-29.

25 V. Brunella, S. A. Jadhav, I. Miletto, G. Berlier, E. Ugazio, S. Sapino, D. Scalarone, React. Funct. Polym. 2016, 98, 31-37. 
26 S. A. Jadhav, V. Brunella, D. Scalarone, G. Berlier, Asian J. Pharm. Sci. 2017, 12, 279-284.

27 E. Ugazio, L. Gastaldi, V. Brunella, D. Scalarone, S. A. Jadhav, S. Oliaro-Bosso, D. Zonari, G. Berlier, I. Miletto, S. Sapino, Int. J. Pharm. 2016, 511, 446- 454.

28 S. A. Jadhav, D. Scalarone, V. Brunella, E. Ugazio, S. Sapino, G. Berlier, Express Polym. Lett. 2017, 11, 96-105.

29 E. Guisasola, A. Baeza, M. Talelli, D. Arcos, M. Vallet-Regí, RSC Adv. 2016, 6, $42510-42516$.

30 E. Yu, A. Lo, L. Jiang, B. Petkus, N. Ileri Ercan, P. Stroeve, Colloids Surf. B Biointerfaces, 2017, 149, 297-300.

31 K. Möller, T. Bein, Chem. Mater. 2017, 29, 371-388.

32 J. Tu, A. L. Boyle, H. Friedrich P. H. H. Bomans, J. Bussmann, N. A. J. M. Sommerdijk, W. Jiskoot, A. Kros, ACS Appl. Mater. Interfaces, 2016, 8, 3221132219.

33 M. Baeza, M. Colilla, M. Vallet-Regí, Expert Opin. Drug Deliv. 2015, 12, 319-337. 
A)
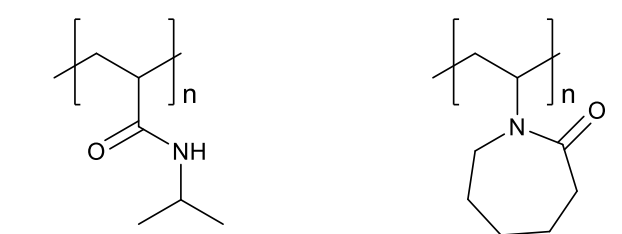

Poly(N-isopropylacrylamide)

Poly(N-vinyl caprolactam)

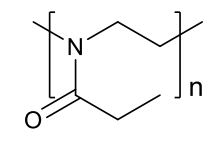

Poly(N-ethyl oxazoline)

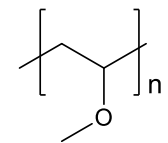

Poly(methyl vinyl ether)

B)

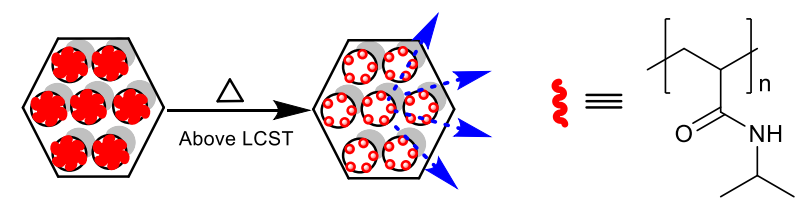

Pores CLOSED

Pores OPENED

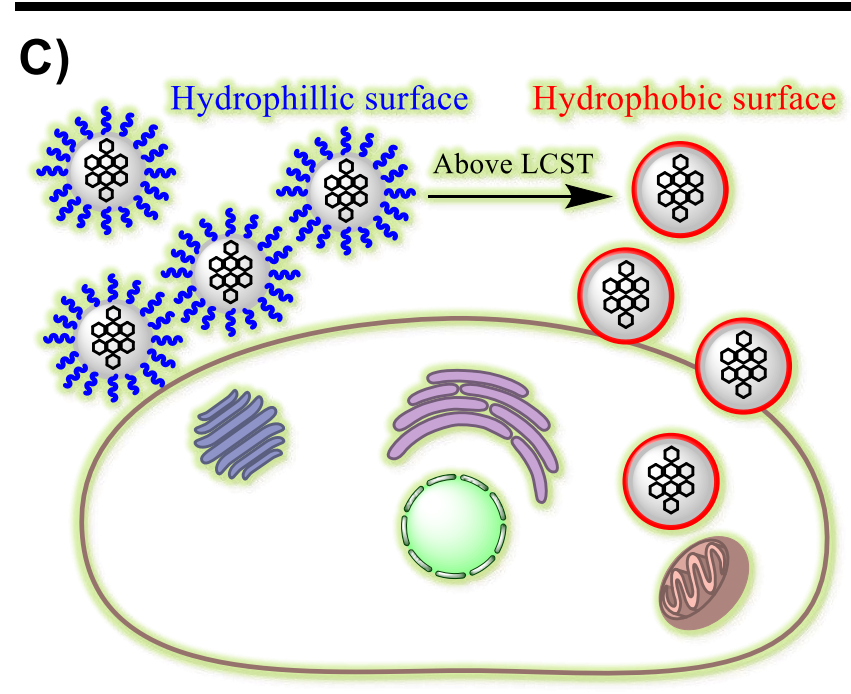

Fig. 1 A) Structures of some common thermoresponsive polymers B) Themoresponsive polymer PNIPAM grafted porous silica showing thermosensitive pore-opening poreclosing mechanism C) Surface property change mediated internalization of thermoresponsive particles above the LCST. 


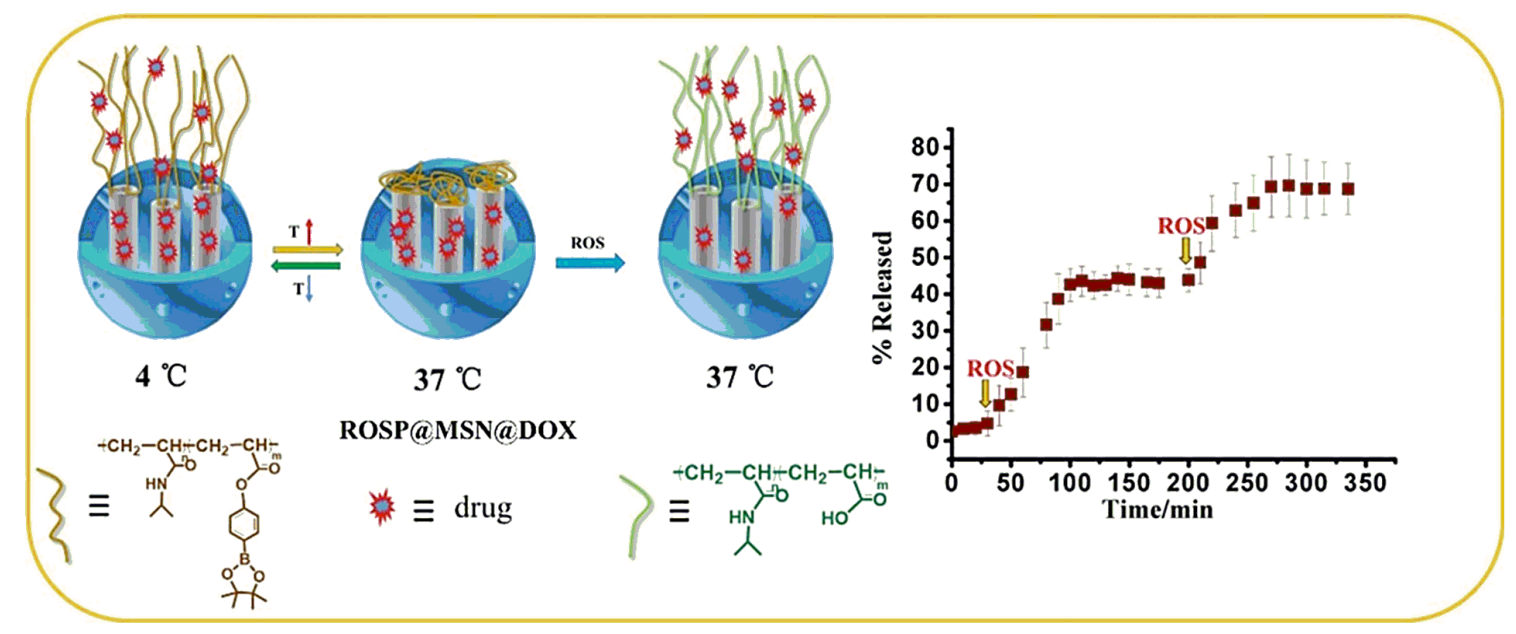

Fig. 2 Thermo and ROS responsive porous silica particles and drug (DOX) release profile from poly (NIPAM-co-AA) generated particles. Reproduced with permission from reference 20. Copyright Elsevier 2018. 

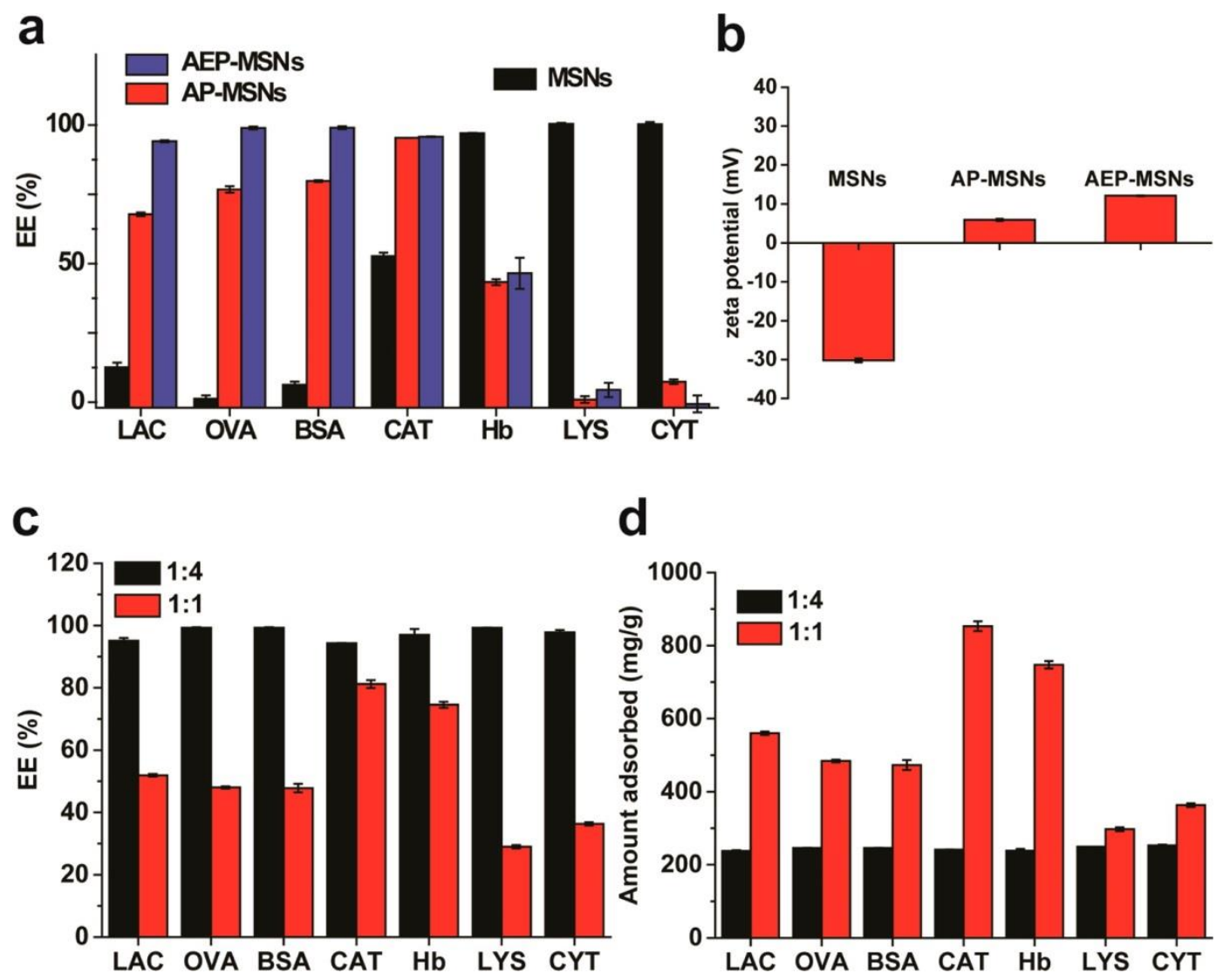

Fig. 3 (a) Encapsulation efficiency (EE\%) of seven proteins in MSNs (black bars), APMSNs (red bars), and AEP-MSNs (blue bars); (b) Zeta potential of MSNs, AP-MSNs, and AEP-MSNs in $1 \mathrm{mM} \mathrm{PB}$, pH 7.4. (c) EE\% of seven proteins (d) Corresponding loading capacity $(\mathrm{mg} / \mathrm{g})$ for all proteins. Reproduced with permission from reference 32 . Copyright ACS 2016. 